\title{
Thermodynamics of Einstein-Born-Infeld black holes in three dimensions
}

\author{
Yun Soo Myung ${ }^{1, a}$, Yong-Wan Kim ${ }^{1, b}$, and Young-Jai Park ${ }^{2, c}$ \\ ${ }^{1}$ Institute of Basic Science and School of Computer Aided Science, \\ Inje University, Gimhae 621-749, Korea \\ ${ }^{2}$ Department of Physics, Sogang University, Seoul 121-742, Korea
}

\begin{abstract}
We show that all thermodynamic quantities of the Einstein-BornInfeld black holes in three dimensions can be obtained from the dilaton and its potential of two dimensional dilaton gravity through the dimensional reduction. These are all between non-rotating uncharged BTZ black hole (NBTZ) and charged BTZ black hole (CBTZ).
\end{abstract}

PACS numbers: 04.70.Dy, 04.60.Kz, 04.20.Jb.

Keywords: Einstein-Born-Infeld black holes; Thermodynamics; Dilaton gravity.

${ }^{a}$ ysmyung@inje.ac.kr

bywkim65@gmail.com

cyjpark@sogang.ac.kr 


\section{Introduction}

In 1934 Born and Infeld proposed a nonlinear electrodynamics giving a finite value for the self-energy of a pointlike charge [1]. Although it became less popular with the introduction of QED, it has been observed that the Born-Infeld action in the presence of scalar fields arises as an effective action governing the dynamics of vector fields on D-branes [2]. For various motivations, extending the Reissner-Nordström (RN) black hole solutions in Einstein-Maxwell theory to the charged black hole solutions in EinsteinBorn-Infeld (EBI) theory with/without a dilaton field has attracted much attention in recent years, for example, see [3, 4, 5]. In particular, Einstein gravity in $(2+1)$ dimensions $[6,7,8]$ has been intensively studied because of the existence of black holes solutions in $(2+1)$-anti de Sitter (AdS) spacetimes $[9,10,11]$, which possess certain features inherent to the $(3+1)$-black holes. Moreover, Cataldo and Garcia had obtained EBI solution for certain range of the parameters mass, charge, cosmological and the Born-Infeld constants, which represent a static circularly symmetric black hole in $(2+1)$ dimensions, although they did not analyze thermodynamic quantities such as heat capacity and free energy [12].

On the other hand, two dimensional (2D) dilaton gravity, which naturally induces the dilaton potential from dimensional reduction, has been used in various situations as an effective description of $4 \mathrm{D}$ gravity after a black hole in string theory has appeared $[13,14]$. In particular, thermodynamics of this black hole has been analyzed by several authors [15, 16, 17, 18]. Another $2 \mathrm{D}$ theories, which were originated from the Jackiw-Teitelboim (JT) theory $[19,20]$, have been also studied [21, 22, 23]. Actually, the 2D dilaton gravity approach is the good $s$-wave approximation to the higher dimensional gravity [24]. Recently, we have introduced the 2D dilaton gravity approach, which completely preserves the thermodynamics of 4D black hole without kinetic term [25], showing that the 2D curvature scalar shows the features of extremal and Davies' points of RN black hole clearly. Very recently, we have resolved the issue of Ruppeiner's geometric approach [26] by analyzing the RN-AdS black holes [27, 28].

It is worth while to point out the literature on the $(2+1)$-EBI gravity in $2 \mathrm{D}$ dilaton gravity approach through the dimensional reduction is rather scarce. Also, it is still believed that $(2+1)$-gravity will provide new insights towards a better understanding of the $(3+1)$-dimensional classical and quantum gravity. In particular, the structure of $(2+1)$-EBI gravity is much simpler than that 
of (3+1)-EBI gravity. As far as we know, it remains a highly nontrivial task to carry out a completely thermodynamic analysis of $(3+1)$-EBI black holes $[3,4]$. In this paper, we address this issue for the EBI black holes in $(2+1)$ dimensions. We will show that all thermodynamic quantities of the EBI black holes can be systematically obtained from the 2D dilaton gravity approach through the dimensional reduction. In addition, we find a possible phase transition between NBTZ and CBTZ.

The organization of this work is as follows. In Sec. 2, we briefly review the procedure to obtain the EBI black hole solution. In Sec. 3, we sketch another approach of 2D dilation gravity to find the EBI black hole solution. In Sec. 4, we investigate all thermodynamic properties of the EBI black holes by comparing those of NBTZ and CBTZ. We summarize our results in Sec. 5. Finally, we derive the ADM mass of EBI black hole by making use of the Hamiltonian analysis in Appendix.

\section{EBI black hole solutions}

Now, let us consider a $(2+1)$-gravity coupled with nonlinear electrodynamics starting from the EBI action

$$
S=\int d^{3} x \sqrt{-g}\left(\frac{R-2 \Lambda}{16 \pi}+L(F)\right),
$$

where

$$
L(F)=\frac{b^{2}}{4 \pi}\left(1-\sqrt{1+\frac{2 F}{b^{2}}}\right) .
$$

Here, the constant $b$ is the Born-Infeld parameter, and $\Lambda$ is the cosmological constant. Notice that this Lagrangian reduces to the Maxwell one in the Maxwell limit of $b^{2} \rightarrow \infty, L(F) \rightarrow-F / 4 \pi$ with $F=\frac{1}{4} F_{\mu \nu} F^{\mu \nu}$. Varying this action with respect to the metric $g_{a b}$ leads to the Einstein equation

$$
\begin{aligned}
& G_{a b}+\Lambda g_{a b}=8 \pi T_{a b}, \\
& T_{a b}=L(F) g_{a b}-F_{a c} F_{b}^{c} L(F),
\end{aligned}
$$

while the variation with respect to the electromagnetic potential $A_{a}$ yields the electromagnetic field equation

$$
\nabla_{a}\left(F^{a b} L_{, F}\right)=0
$$

Here the Latin index $a, b, c \cdots$ denote $3 \mathrm{D}$ spacetimes and $L_{, F}$ represents the derivative of $L(F)$ with respect to $F$. In order to obtain the EBI black hole 
solution, we consider a static circular metric in $(2+1)$ dimensions as follows

$$
d s_{3 D}^{2}=g_{a b} d x^{a} d x^{b}=-U(r) d t^{2}+\frac{d r^{2}}{U(r)}+r^{2} d \theta^{2}
$$

where $U(r)$ is the unknown metric function. Here we choose the electric field only to be

$$
F_{a b}=E(r)\left(\delta_{a}^{t} \delta_{b}^{r}-\delta_{a}^{r} \delta_{b}^{t}\right)
$$

Since the invariant is given by $F=-E^{2}(r) / 2$, from Eq. (5) we obtain the solution for electric field

$$
E(r)=\frac{Q}{\sqrt{r^{2}+Q^{2} / b^{2}}} .
$$

Here $Q$ is an integration constant to represent the charge. In the Maxwell limit, we recover a correct form of $E_{M L}=Q / r$ in $(2+1)$ dimensions. Hence the electric field of BI theory $E(r)$ in Eq. (8) represents the smeared version of $E_{M L}=Q / r$. Plugging the electric configuration into the $(\theta, \theta)$-component of Einstein equation (3) with $\Lambda=-1 / l^{2}$ leads to

$$
U_{, r}=\frac{2 r}{l^{2}}-\frac{4 Q^{2}}{\sqrt{r^{2}+Q^{2} / b^{2}}}-4 b^{2} r\left(\frac{r}{\sqrt{r^{2}+Q^{2} / b^{2}}}-1\right) .
$$

Then one finds the metric function after the integration of $\int_{r_{0}}^{r} U(\tilde{r})_{, \tilde{r}} d \tilde{r}$

$$
\begin{aligned}
U(r) & =-M+\frac{r^{2}}{l^{2}}+2 b^{2} r\left(r-\sqrt{r^{2}+\frac{Q^{2}}{b^{2}}}\right)-2 Q^{2} \ln \left[r+\sqrt{r^{2}+\frac{Q^{2}}{b^{2}}}\right](10) \\
& +2 Q^{2} \ln \left[l+\sqrt{l^{2}+\frac{Q^{2}}{b^{2}}}\right]-2 b^{2} l\left[l-\sqrt{l^{2}+\frac{Q^{2}}{b^{2}}}\right] .
\end{aligned}
$$

Here we choose $r_{0}=l$ for our purpose. This solution is different from the old solution (20) in Ref. [12] by two terms in the second line. In fact, the old solution has some ambiguity. In the Maxwell limit of $b^{2} \rightarrow \infty$, it could not recover the charged BTZ black hole solution [11]

$$
U_{C B T Z}(r)=-M+\frac{r^{2}}{l^{2}}-2 Q^{2} \ln \left(\frac{r}{l}\right)
$$

Also, counting the mass dimensions of $[Q]=0,[b]=1$, and $[l]=-1$, we find that the log-term in the first line has " -1 " dimension, which contradicts to "zero" dimension. This is because they did not evaluate the lower bound correctly, like as $\int^{r} U(\tilde{r})_{, \tilde{r}} d \tilde{r}$. However, these two disappear in our solution 
because we evaluate it correctly, as $\int_{r_{0}}^{r} U(\tilde{r})_{, \tilde{r}} d \tilde{r}$ with $r_{0}=l$. The last term in the second line comes from the third term of the first line with $r=r_{0}$, while the first term in the second line gives a correct zero dimension when combining it with the last term in the first line. The relevant parameters for EBI black holes are $Q$ and $b$. The mass of EBI black holes is obtained from $U(r)=0$ as the function of horizon radius $r$,

$$
\begin{aligned}
M(r) & =\frac{r^{2}}{l^{2}}+2 b^{2} r\left(r-\sqrt{r^{2}+\frac{Q^{2}}{b^{2}}}\right)-2 Q^{2} \ln \left[r+\sqrt{r^{2}+\frac{Q^{2}}{b^{2}}}\right] \\
& +2 Q^{2} \ln \left[l+\sqrt{l^{2}+\frac{Q^{2}}{b^{2}}}\right]-2 b^{2} l\left[l-\sqrt{l^{2}+\frac{Q^{2}}{b^{2}}}\right] .
\end{aligned}
$$

\section{$3 \quad 2 \mathrm{D}$ dilaton gravity approach}

Now, we are in a position to derive the EBI black hole solution $U(r)$ in Eq. (10) from the dilaton gravity approach.

Assuming $\mathcal{M}_{3}=\mathcal{M}_{2} \times S^{1}$ for our purpose, we perform a Kaluza-Klein reduction

$$
d s_{3 D}^{2}=g_{a b} d x^{a} d x^{b}=\bar{g}_{\mu \nu} d \bar{x}^{\mu} d \bar{x}^{\nu}+\phi^{2}(\bar{x}) d \theta^{2},
$$

where $\phi$ represents the radius $r$ of circle $S^{1}$. Here the Greek indices $\mu, \nu, \cdots$ represent the two-dimensional spacetimes. After the dimensional reduction by integrating Eq. (1) over $S^{1}$, the effective 2D dilaton action is given by

$$
S_{2 D}=2 \pi \int d^{2} \bar{x} \sqrt{-\bar{g}} \phi\left(\frac{\bar{R}_{2}-2 \Lambda}{16 \pi}+L(F)\right) .
$$

Here, $\bar{R}_{2}$ is the 2D Ricci scalar. Note that since there are no kinetic term for dilaton, it is not necessary to perform conformal transformation to find the black hole thermodynamics. This contrasts to the cases in higher dimensions than three $[25,27,28]$

For simplicity, by omitting 'bar', the effective field equations of the EBI theory in two dimensions are given by

$$
\begin{aligned}
\nabla^{\nu}\left(\frac{\sqrt{-g} \phi F_{\mu \nu}}{\sqrt{1+\frac{2 F}{b^{2}}}}\right) & =0, \\
\nabla^{2} \phi+2 \phi \Lambda & =16 \pi \phi\left(L(F)-\frac{\partial L(F)}{\partial g^{\mu \nu}} g^{\mu \nu}\right), \\
R_{2}-2 \Lambda & =-16 \pi L(F) .
\end{aligned}
$$


We note that $R_{2}$ differs from the $(2+1)$ dimensional Ricci scalar $R$ in Eq. (1). Even though $R$ is singular at $r=0, R_{2}$ is regular because it is the 2D Ricci scalar. It could be de Sitter $\left(R_{2}>0\right)$, flat $\left(R_{2}=0\right)$, and anti de Sitter spacetimes $\left(R_{2}<0\right)$. Actually $R_{2}$ is related to thermodynamic quantity, heat capacity (See Eqs. (21) and (43)). Now, as a concrete solution of EBI dynamical equations, we present a static self-consistent solution in the 2D dilaton approach. Then the restricted electric field becomes to be $F_{\mu \nu}=E(r)\left(\delta_{\mu}^{t} \delta_{\nu}^{r}-\delta_{\mu}^{r} \delta_{\nu}^{t}\right)$. Since the invariant is given by $2 F=-E^{2}(r)$ from the electromagnetic field equations, we obtain the solution for electric field as follows

$$
E(\phi)=\frac{Q}{\sqrt{\phi^{2}+Q^{2} / b^{2}}} .
$$

Considering

$$
\begin{aligned}
& L(F)=\frac{b^{2}}{4 \pi}\left(1-\frac{b \phi}{\sqrt{b^{2} \phi^{2}+Q^{2}}}\right), \\
& \frac{\partial L(F)}{\partial g^{\mu \nu}}=-\frac{F_{\mu \rho} F_{\nu}{ }^{\rho}}{8 \pi \sqrt{1+2 F / b^{2}}},
\end{aligned}
$$

equations (6) and (7) take the forms

$$
\begin{aligned}
& \nabla^{2} \phi=V(\phi)=-2 \phi \Lambda+4 b^{2}\left(\phi-\sqrt{\phi^{2}+Q^{2} / b^{2}}\right) \\
& R_{2}=-V^{\prime}(\phi)=2 \Lambda+4 b^{2}\left(\frac{\phi}{\sqrt{\phi^{2}+Q^{2} / b^{2}}}-1\right) .
\end{aligned}
$$

These are exactly the same equations derived from the $2 \mathrm{D}$ dilaton gravity with potential $V(\phi)$,

$$
\bar{I}_{2}=\int_{\mathcal{M}_{2}} d x d t \sqrt{-g}\left[\phi R_{2}+V(\phi)\right]
$$

In order to solve the above two equations, we introduce the Schwarzschildtype gauge for $g_{\mu \nu}$ as

$$
g_{\mu \nu}=\operatorname{diag}\left(-f, f^{-1}\right) .
$$

Then, its curvature scalar takes the form

$$
R_{2}=-f^{\prime \prime}
$$

where the prime "l" denotes the derivative with respect to $x$. We note from Eq. (13) that the dilaton is independent of time $t(\phi=\phi(x))$. Eqs. (21) and (22) reduce to

$$
f \phi^{\prime \prime}+f^{\prime} \phi^{\prime}=V(\phi), f^{\prime \prime}=\frac{d V(\phi)}{d \phi} .
$$


In addition, we have the kinetic term for $\phi$

$$
(\nabla \phi)^{2}=f\left(\phi^{\prime}\right)^{2} .
$$

If one chooses the linear dilaton as the solution

$$
\phi=x,
$$

then Eq. (26) leads to

$$
f^{\prime}=V(\phi), f^{\prime \prime}=V^{\prime}(\phi),
$$

which imply that the latter is just a redundant relation. Then, we obtain the solution to Eqs. (21) and (22) as

$$
d s_{2 D}^{2}=g_{\mu \nu} d x^{\mu} d x^{\nu}=-f(\phi) d t^{2}+\frac{1}{f(\phi)} d \phi^{2} .
$$

Here the metric function $f(\phi)$ is given by

$$
f(\phi)=J(\phi)-\mathcal{C},
$$

where $J(\phi)$ is the integration of $V(\phi)$ with a reference point $\phi_{0}=l$ given by

$$
J(\phi)=\int_{\phi_{0}}^{\phi} V(\tilde{\phi}) d \tilde{\phi} .
$$

Also $\mathcal{C}$ is a coordinate-invariant constant of integration, which will be identified with the mass $M$ of the EBI black hole by using the Hamiltonian approach in Appendix.

Taking into account $\Lambda=-1 / l^{2}$, we obtain essential quantities for describing thermodynamics of EBI black hole: dilaton potential $V$, its derivative $V^{\prime}$, and its integration $J$ as

$$
\begin{aligned}
V(\phi) & =\frac{2 \phi}{l^{2}}+4 b^{2}\left(\phi-\sqrt{\phi^{2}+Q^{2} / b^{2}}\right) \\
V^{\prime}(\phi) & =\frac{2}{l^{2}}+4 b^{2}\left(1-\frac{\phi}{\sqrt{\phi^{2}+Q^{2} / b^{2}}}\right), \\
J(\phi) & =\frac{\phi^{2}}{l^{2}}+2 b^{2} \phi\left(\phi-\sqrt{\phi^{2}+\frac{Q^{2}}{b^{2}}}\right)-2 Q^{2} \ln \left[\phi+\sqrt{\phi^{2}+\frac{Q^{2}}{b^{2}}}\right] \\
& +2 Q^{2} \ln \left[l+\sqrt{l^{2}+\frac{Q^{2}}{b^{2}}}\right]-2 b^{2} l\left[l-\sqrt{l^{2}+\frac{Q^{2}}{b^{2}}}\right] .
\end{aligned}
$$


Here we confirm the relation of metric function between $U(r)$ and $f(\phi)$ for setting $\phi=r$ as follows

$$
U(r)=f(r),
$$

which means that a 2D dilaton gravity approach gives us the EBI black hole solution too. We note that the mass of EBI black hole is given by $M(r)=$ $J(\phi)=\mathcal{C}$ when considering Eqs.(12) and (35). Hereafter we call this black hole as the Born-Infeld-Banados-Teitelboim-Zanelli black hole (BIBTZ). Here we mention the two limiting cases: uncharged limit of $Q=0$ and Maxwell limit of $b \rightarrow \infty$ with $Q \neq 0$. The former provides the non-rotating BTZ black hole (NBTZ) described by [29]

$$
V_{N B T Z}=\frac{2 \phi}{l^{2}}, V_{N B T Z}^{\prime}=\frac{2}{l^{2}}, J_{N B T Z}=\frac{\phi^{2}}{l^{2}}
$$

and the latter gives the charged BTZ black hole (CBTZ) described by

$$
V_{C B T Z}=\frac{2 \phi}{l^{2}}-\frac{2 Q^{2}}{\phi}, V_{C B T Z}^{\prime}=\frac{2}{l^{2}}+\frac{2 Q^{2}}{\phi^{2}}, J_{C B T Z}=\frac{\phi^{2}}{l^{2}}-2 Q^{2} \ln \left(\frac{\phi}{l}\right) .
$$

Their metric functions are given by

$$
\begin{aligned}
f_{N B T Z}(\phi) & =-M+J_{N B T Z}=-M+\frac{\phi^{2}}{l^{2}}, \\
f_{C B T Z}(\phi) & =-M+J_{C T B Z}=-M+\frac{\phi^{2}}{l^{2}}-2 Q^{2} \ln \left(\frac{\phi}{l}\right) .
\end{aligned}
$$

This is why we use "BTZ" in BIBTZ. We note that these two limits exist for the whole region of $\phi$.

\section{Thermodynamics of EBI black holes}

In order to describe thermodynamics of BIBTZ in terms of 2D dilaton quantities of Eqs.(33)-(35), we have to know the extremal BIBTZ. From Eq.(26) with the extremal condition $f^{\prime}(x)=0$ (equivalently, $V(\phi)=0$ ), we have the extremal value

$$
\phi_{e}=\frac{2 b l^{2} Q}{\sqrt{1+4 b^{2} l^{2}}}
$$

Obviously, we have two limiting cases: $\phi_{e}=0$ for NBTZ and $\phi_{e}=Q l$ for CBTZ. Inserting this into $J(\phi)$, we have the extremal mass $M_{e}$

$$
\begin{aligned}
M_{e}= & -2 Q^{2} \ln \left[\frac{Q}{b} \sqrt{1+4 b^{2} l^{2}}\right]+2 Q^{2} \ln \left[l+\sqrt{l^{2}+Q^{2} / b^{2}}\right] \\
& -2 b^{2} l\left[l-\sqrt{l^{2}+Q^{2} / b^{2}}\right] .
\end{aligned}
$$



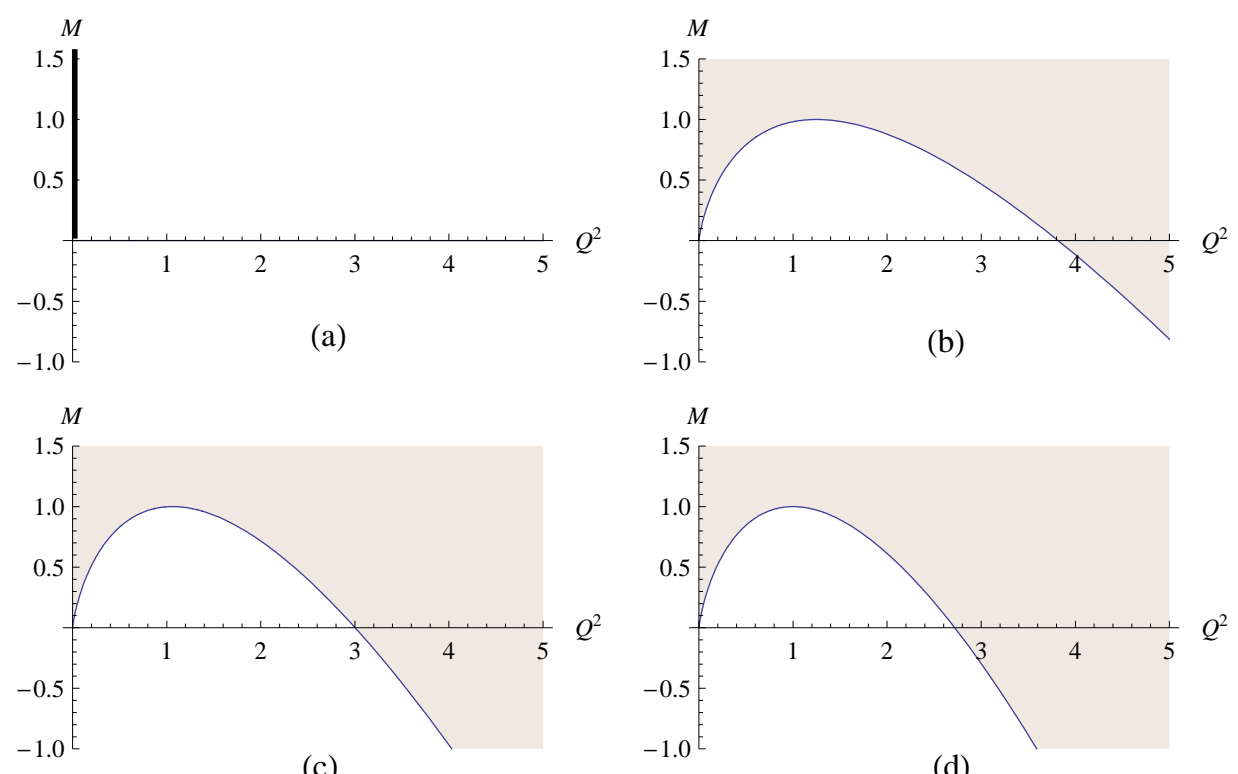

(c)

(d)

Figure 1: Regions of mass-charge plane with $l=10$. (a) $Q=0$ (NBTZ) (b) $b=0.1, Q=1$ (c) $b=0.2, Q=1$ (d) $b=100, Q=1$ (CBTZ). Black holes exist on the positive $M$-axis for NBTZ and the shaded areas for all BIBTZ including CBTZ. Extremal black holes are $(0,0)$ point for NBTZ, while these are boundary curves for BIBTZ $(b=0.1,0.2,100)$. CBTZ has the minimal NS region (white area).

For $M>M_{e}$, there exist two horizons $\phi=\phi_{ \pm}$, while for $M=M_{e}$, we obtain the degenerate horizon $\phi=\phi_{e}$. For thermodynamic analysis, we need the outer horizon of $\phi_{+}$and hereafter we omit "+". On the other hand, for $M<M_{e}$, there exists no horizon, or naked singularity (NS). In Fig. 1, we depict the behaviors of $M$ versus $Q^{2}$ to understand the BIBTZ. As is well known, the extremal point of NBTZ is just zero (massless BTZ black hole) and the whole region belongs to NS (Fig. 1a), while in the Maxwell limit (CBTZ: Fig. 1d of $b=100$ ), we find the minimal NS region [11]. On the other hand, there is the NS region of BIBTZ between two limiting cases. We note that if the electric charge $Q$ is large, the BIBTZ exits even for negative masses. This is in sharp contrast with what happens in four dimensions. In this sense, the BIBTZ including CBTZ is problematic. Also we mention that the global structure of BIBTZ, Penrose diagram is similar to that of CBTZ because BIBTZ has two horizons in asymptotically AdS spacetimes.

In order to explore thermodynamics of BIBTZ, from Eqs. (33)-(35), we obtain the thermodynamic functions of Hawking temperature $T(\phi)$, heat 


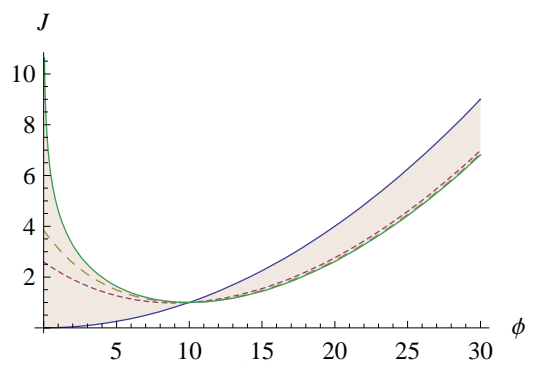

(a)

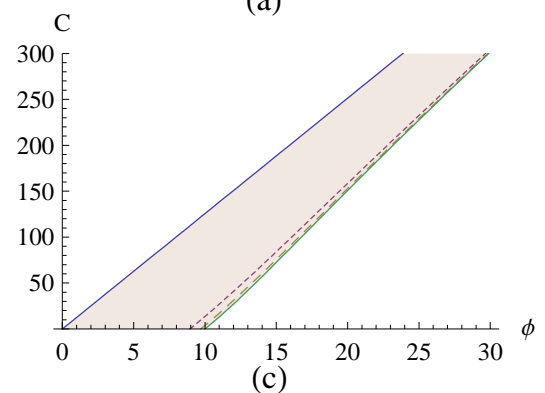

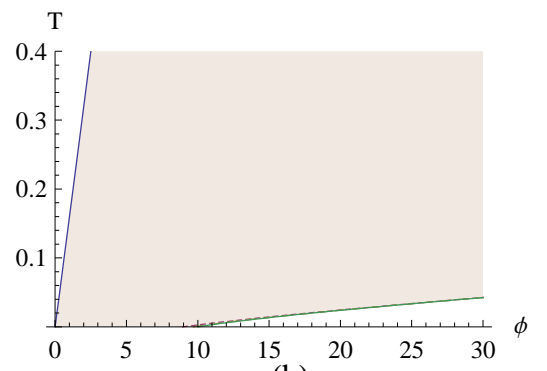

(b)

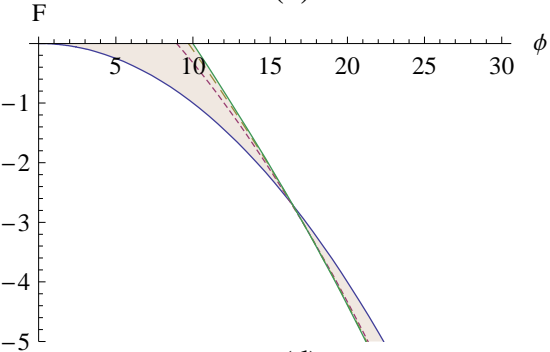

(d)

Figure 2: Thermodynamic quantities for BIBTZ (shaded areas) with $Q=$ $1, l=10$. (a) Mass graph $J(\phi)$. (b) Temperature graph $T(\phi)$. (c) Heat capacity graph $C(\phi)$. (d) Free energy $F(\phi)$. All inner curves denote $b=0.1$ next-nearest and $b=0.2$ nearest to $b=100$ (CBTZ).

capacity $C(\phi)$, and free energy $F(\phi)$ as follows:

$$
\begin{aligned}
T(\phi) & =\frac{V(\phi)}{4 \pi}, \\
C(\phi) & =4 \pi\left(\frac{V(\phi)}{V^{\prime}(\phi)}\right), \\
F(\phi) & =J(\phi)-J\left(\phi_{e}\right)-\phi V(\phi) .
\end{aligned}
$$

Note that defining the free energy, we use the extremal case as the ground state because we are working with the canonical ensemble of fixed-charge $Q[30]$.

In the Maxwell limit (CBTZ) [11], we have the thermodynamic functions as follows

$$
\begin{aligned}
T_{C B T Z}(\phi) & =\frac{1}{2 \pi}\left(\frac{\phi}{l^{2}}-\frac{Q^{2}}{\phi}\right) \\
C_{C B T Z}(\phi) & =4 \pi\left(\frac{\frac{\phi}{l^{2}}-\frac{Q^{2}}{\phi}}{\frac{1}{l^{2}}+\frac{Q^{2}}{\phi^{2}}}\right) \\
F_{C B T Z}(\phi) & =-\frac{\phi^{2}}{l^{2}}+Q^{2}-2 Q^{2} \ln \left(\frac{\phi}{Q l}\right) .
\end{aligned}
$$


On the other hand, in the uncharged limit (NBTZ), the corresponding thermodynamic functions are given by

$$
T_{N B T Z}(\phi)=\frac{1}{2 \pi} \frac{\phi}{l^{2}}, \quad C_{N B T Z}(\phi)=4 \pi \phi, \quad F_{N B T Z}(\phi)=-\frac{\phi^{2}}{l^{2}} .
$$

Fig. 2a shows mass graph $J(\phi)$ for BIBTZ. The shaded region is for BIBTZ. The upper boundary corresponds to $\mathrm{CBTZ}(b=100)$ in the region of $0 \leq$ $\phi \leq Q l$ and $\operatorname{NBTZ}(Q=0)$ in the region of $\phi \geq Q l$. The lower boundary corresponds to NBTZ in the region of $0 \leq \phi \leq Q l$ and CBTZ in the region of $\phi \geq Q l$. The two masses meet at $\phi=\phi_{e}$ for CBTZ. This picture implies clearly that BIBTZ has the extremal black hole as the minimum point of $d J / d \phi=0$.

Temperature graph $T(\phi)$ is depicted in Fig. 2b. The shaded region is for BIBTZ. The left boundary corresponds to NBTZ and the bottom line of $0 \leq \phi \leq Q l$ is for the extremal BIBTZ. The sloping line on the bottom means the temperature of CBTZ. Temperature of BIBTZ has the similar behavior: $T=0$ as the extremal point and a monotonically increasing function.

Fig. 2c indicates heat capacity graph $C(\phi)$ for BIBTZ. This behavior is the same as that of the entropy $S(\phi)$. The shaded region is for BIBTZ. The left boundary corresponds to NBTZ and the bottom line is for the extremal BIBTZ. The right boundary denotes the heat capacity of CBTZ. This means that all BIBTZ are thermodynamically stable because of their positive heat capacity.

Finally, free energy $F(\phi)$ is depicted in Fig. 3d. The shaded region is for BIBTZ. This graph shows an interesting point at $\phi=\phi_{t}=l e^{1 / 2}$ which is found from the condition of $F_{N B T Z}=F_{C B T Z}$. The left boundary corresponds to NBTZ in the region of $0 \leq \phi \leq \phi_{t}$ and CBTZ in the region of $\phi \geq \phi_{t}$. The right boundary corresponds to CBTZ in the region of $Q l \leq \phi \leq \phi_{t}$ and NBTZ in the region of $\phi \geq \phi_{t}$. This may imply a second-order phase transition between NBTZ and CBTZ [31, 32].

\section{Summary}

We have systematically obtained all thermodynamic quantities of the EinsteinBorn-Infeld black holes in three dimensions from the dilaton and its potential of 2D dilaton gravity. This was performed through the dimensional reduction by comparing those of the Maxwell and BTZ black holes. These are all between non-rotating uncharged black hole (NBTZ) and charged black hole (CBTZ). Actually, the nonlinearity of BI action weakens the divergence of 
the curvature scalar of CBTZ [33] and could connect the regular NBTZ to singular CBTZ. Hence we clearly understand the role of BI action in constructing the 3D EBI black holes (BIBTZ) in the sense that the BIBTZ plays the role of a toy model for higher dimensional EBI black holes.

In addition, we have observed from free energy that the second-order phase transition may occur between NBTZ and CBTZ [31, 32]. For $Q l<$ $\phi<\phi_{t}$, the NBTZ configuration is more favorable than the CBTZ, while for $\phi>\phi_{t}$, the CBTZ configuration is more favorable than the NBTZ. This means that for $Q l<\phi<\phi_{t}$, the ground state is the NBTZ, whereas for $\phi>\phi_{t}$, the ground state is chosen to be the CBTZ.

\section{Appendix: ADM mass from the Hamiltonian analysis}

In this appendix, we show explicitly that the integration constant $\mathcal{C}$ in Eq. (31) is just the Arnowitt-Deser-Missner (ADM) mass [34] through the Hamiltonian analysis by using the notation and convention of Refs. [35, 36, 37].

Let us start with the different metric given by the ADM-parametrization

$$
d s_{A D M}^{2} \equiv h_{\mu \nu} d x^{\mu} d x^{\nu}=e^{2 \rho}\left[-u^{2} d t^{2}+(d x+v d t)^{2}\right],
$$

where $\rho, u$, and $v$ are functions of the coordinates $(t, x)$. In terms of the parametrization (A1), the action of $2 \mathrm{D}$ dilaton gravity in Eq.(23) is expressed as

$$
\begin{aligned}
\bar{I}_{2} & =\int d t \int_{\sigma_{-}}^{\sigma_{+}} d x\left[\frac{\dot{\phi}}{u}\left(2 v \rho^{\prime}+2 v^{\prime}-2 \dot{\rho}\right)\right. \\
& \left.+\frac{\phi^{\prime}}{u}\left(2 u u^{\prime}-2 v v^{\prime}+2 v \dot{\rho}+2 u^{2} \rho^{\prime}-2 v^{2} \rho^{\prime}\right)+u e^{2 \rho} V(\phi)\right],
\end{aligned}
$$

where the overdots and primes denote differentiation with respect to time and space, respectively, while $\sigma_{+}\left(\sigma_{-}\right)$is the outer (inner) spatial boundary.

Following the Hamiltonian formulation, we have the canonical momenta

$$
\begin{aligned}
\pi_{\phi} & =\frac{2}{u}\left(-\dot{\rho}+v \rho^{\prime}+v^{\prime}\right), \\
\pi_{\rho} & =\frac{2}{u}\left(-\dot{\phi}+v \phi^{\prime}\right), \\
\pi_{u} & =0 \\
\pi_{v} & =0
\end{aligned}
$$


and the canonical Hamiltonian

$$
H_{c}=\int d x(v \mathcal{F}+u \mathcal{G})
$$

where $\mathcal{F}$ and $\mathcal{G}$ are defined as

$$
\begin{aligned}
\mathcal{F} & =\rho^{\prime} \pi_{\rho}+\phi^{\prime} \pi_{\phi}-\pi_{\rho}^{\prime} \approx 0, \\
\mathcal{G} & =2 \phi^{\prime \prime}-2 \phi^{\prime} \rho^{\prime}-\frac{1}{2} \pi_{\rho} \pi_{\phi}-e^{2 \rho} V(\phi) \approx 0 .
\end{aligned}
$$

Here, $\approx$ denotes that it is weakly vanishing on the constraint surfaces in the sense of Dirac formulation [38]. Note that $\pi_{u}, \pi_{v}(\mathcal{F}, \mathcal{G})$ are primary (secondary) constraints, and $u, v$ play the role of the Lagrange multipliers.

We consider the linear combination of the constraints as

$$
\mathcal{E} \equiv-e^{-2 \rho}\left(\phi^{\prime} \mathcal{G}+\frac{1}{2} \pi_{\rho} \mathcal{F}\right) \approx 0
$$

Since $\mathcal{E}$ commutes with both the constraints $\mathcal{F}$ and $\mathcal{G}$, it is a physical quantity in the Dirac's sense. Moreover, it can be rewritten by a spatial derivative as

$$
\mathcal{E}=\frac{\partial}{\partial x}\left[e^{-2 \rho}\left(\frac{1}{4} \pi_{\rho}^{2}-\phi^{2}\right)+J(\phi)\right] \equiv \frac{\partial \mathcal{M}}{\partial x} .
$$

Here we have defined the terms in the square bracket as the mass observable $\mathcal{M}$.

At this stage, we show that $\mathcal{M}$ is the constant of $\mathcal{C}$ in Eq.(31) on the constraint surface. The constraint surface implies that the off-diagonal form of $h_{\mu \nu}$ in Eq.(A1) reduces to the diagonal form of $g_{\mu \nu}$ in Eq.(30). In this case, we have the connection

$$
\left.\mathcal{M}\right|_{c s}=-(\nabla \phi)^{2}+J(\phi)=\mathcal{C}
$$

where we used Eqs.(27) and (31).

Making use of the linear combination of the constraints, we can further rewrite the canonical Hamiltonian as

$$
H_{c}=\int d x\left(-\tilde{u} \mathcal{M}^{\prime}+\tilde{v} \mathcal{F}\right)+H_{+}-H_{-},
$$

where $\tilde{u}, \tilde{v}$ are given by

$$
\tilde{u}=\frac{u}{\phi^{\prime}} e^{2 \rho}, \quad \tilde{v}=v-\frac{u}{2 \phi^{\prime}} \pi_{\rho} .
$$

Here, $H_{+}, H_{-}$are boundary terms which make the surface terms in the variation of $H_{c}$ vanish for a given boundary condition. As a boundary condition, 
if we choose the value of the dilaton fixed at $u_{+}$and make it to be time independent, the time independence of the dilaton implies that $\tilde{v}_{+}=0$, or, equivalently, $\left.\pi_{\rho}\right|_{\sigma_{+}}=2 v \phi^{\prime} /\left.u\right|_{\sigma_{+}}$.

As a result, we obtain the canonical Hamiltonian given by the first term in Eq. (A13) and $H_{+}$. The variation of the resulting Hamiltonian is simply written as

$$
\delta H_{+}(\mathcal{M})=\left.\tilde{u} \delta \mathcal{M}\right|_{\sigma_{+}} .
$$

By solving $\mathcal{M}$ and $\left.\pi_{\rho}\right|_{\sigma_{+}}$at $u_{+}$in terms of $\tilde{u}$ as

$$
\tilde{u}^{2}=\frac{h_{t t}}{\mathcal{M}-J\left(\phi_{+}\right)},
$$

Eq. (A15) can be easily integrated to give

$$
H_{+}(\mathcal{M})=2 \sqrt{-h_{t t} J\left(\phi_{+}\right)}\left(1-\sqrt{1-\frac{\mathcal{M}}{J\left(\phi_{+}\right)}}\right) .
$$

Note that the integration constant is fixed so that $H_{+} \rightarrow 0$ as $\mathcal{M} \rightarrow 0$, and $h_{t t}$ is also fixed at the boundary. $H_{+}(\mathcal{M})$ is the dilaton gravity analogue of the Brown-York quasi-local energy [39].

Finally, as $\phi_{+}$goes to the infinity, one has

$$
H_{+}(\mathcal{M}) \rightarrow \sqrt{\frac{-h_{t t}}{J\left(\phi_{+}\right)}} \mathcal{M} .
$$

Therefore, this gives to the ADM mass, $H_{A D M} \rightarrow \mathcal{M}$, providing the physical metric is normalized to one at spatial infinity [37]. The constant of $\sqrt{\frac{-h_{t+}}{J\left(\phi_{+}\right)}}$ may be related to the symmetry of the $2 \mathrm{D}$ dilaton gravity. The form of the metric (30) is preserved by the following global rescalings: $\phi \rightarrow a \phi, f \rightarrow$ $a^{2} f, t \rightarrow t / a$. Then, the mass seems to be $a^{2} \mathcal{C}$, and not $\mathcal{C}$. Actually, any function of $\mathcal{C}$ can be called mass basing on the only relevant argument that $\mathcal{C}$ is coordinate invariant. In general, the mass of a black hole is the value of the Hamiltonian calculated for the black hole solution, and this calculation involved a careful analysis of asymptotic behavior of the metric. However, the ADM mass of $H_{+}(\mathcal{M})$ is not determined completely even after the Hamiltonian calculation.

\section{Acknowledgments}

Y. S. Myung was supported by the Korea Research Foundation (KRF-2006311-C00249) funded by the Korea Government (MOEHRD). Y.-W. Kim was 
supported by the Korea Research Foundation Grant funded by Korea Government (MOEHRD): KRF-2007-359-C00007. Y.-J. Park was supported by the Korea Science and Engineering Foundation (KOSEF) grant funded by the Korea government (MOST) (R01-2007-000-20062-0).

\section{References}

[1] M. Born and L. Infeld, Proc. R. Soc. London A 144, 425 (1934).

[2] A.A. Tseytlin, Nucl. Phys. B 276, 391 (1986) [Erratum-ibid. B 291, 876 (1987)].

[3] D.L. Wiltshire, Phys. Rev. D 38, 2445 (1988); D. A. Rasheed, "Nonlinear electrodynamics: Zeroth and first laws of black hole mechanics," arXiv:hep-th/9702087;

T. Tamaki and T. Torii, Phys. Rev. D 62, 061501 (2000) [arXiv:grqc/0004071];

N. Breton, Phys. Rev. D 67, 124004 (2003) [arXiv:hep-th/0301254];

S. Fernando and D. Krug, Gen. Relativ. Gravit. 35, 129 (2003) [arXiv:hep-th/0306120];

M. Aiello, R. Ferraro, and G. Giribet, Phys. Rev. D 70, 104014 (2004) [arXiv:gr-qc/0408078].

[4] T. K. Dey, Phys. Lett. B 595, 484 (2004) [arXiv:hep-th/0406169];

S. Fernando, Phys. Rev. D 74, 104032 (2006) [arXiv:hep-th/0608040].

[5] A. Sheykhi, Phys. Lett. B 662, 7 (2008) [arXiv:0710.3827 [hep-th]].

[6] S. Carlip, J. Korean Phys. Soc. 28, S447 (1995) [arXiv:gr-qc/9503024].

[7] R. Mann, "Lower dimensional black holes: Inside and out," arXiv:grqc/9501038.

[8] V. Frolov, S. Hendy, A.L. Larsen, Nucl. Phys. B 468, 336 (1996) [arXiv:hep-th/9602033].

[9] M. Banados, C. Teitelboim, J. Zanelli, Phys. Rev. Lett. 69, 1849 (1992) [arXiv:hep-th/9204099].

[10] M. Banados, M. Henneaux, C. Teitelboim, and J. Zanelli, Phys. Rev. D 48, 1506 (1993) [arXiv:gr-qc/9302012]. 
[11] C. Martinez, C. Teitelboim and J. Zanelli, Phys. Rev. D 61, 104013 (2000) [arXiv:hep-th/9912259].

[12] M. Cataldo and A. Carcia, Phys. Lett. B 456, 28 (1999) [arXiv:hepth/9903257].

[13] E. Witten, Phys. Rev. D 44, 314 (1991).

[14] G. Mandal, A. M. Sengupta, and S. R. Wadia, Mod. Phys. Lett. A 6, 1685 (1991).

[15] C. G. Callan, S. B. Giddings, J. A. Harvey, and A. Strominger, Phys. Rev. D 45, R1005 (1992) [arXiv:hep-th/9111056].

[16] J. G. Russo, L. Susskind, L. Thorlacius, Phys. Lett. B 292, 13 (1992).

[17] V. P. Frolov, Phys. Rev. D 46, 5383 (1992).

[18] D. Grumiller, W. Kummer, D. V. Vassilevich, Phys. Rept. 369, 327 (2002).

[19] R. Jackiw, in Quantum Theory of Gravity, ed. S. M. Christensen (Hilger, Bristol, 1984).

[20] C. Teitelboim, in Quantum Theory of Gravity, ed. S. M. Christensen (Hilger, Bristol, 1984).

[21] M. Henneaux, Phys. Rev. Lett. 54, 959 (1985).

[22] R. B. Mann, D. Robbins, and T. Ohta, Phys. Rev. Lett. 82, 3738 (1999) [arXiv:gr-qc/9811061].

[23] A. Fabbri, D. J. Navarro, and J. Navarro-Salas, Nucl. Phys. B 595, 381 (2001).

[24] S. Nojiri and S. D. Odintsov, Phys. Lett. B 463, 57 (1999) [arXiv:hepth/9904146].

[25] Y.S. Myung, Y.-W. Kim and Y.-J. Park, Mod. Phys. Lett. A 23, 91 (2008) [arXiv:0707.3314 [gr-qc]]; Y.S. Myung, Y.-W. Kim and Y.-J. Park, "Thermodynamics of regular black hole", arXiv:0708.3145 [gr-qc]; Y.S. Myung, Mod. Phys. Lett. A 23, 667 (2008) [arXiv:0710.2568 [grqc]]. 
[26] G. Ruppeiner, "Black Holes: Fermions at the Extremal Limit?", arXiv:0711.4328 [gr-qc]; "Thermodynamic curvature and phase transitions in Kerr-Newman black holes", arXiv:0802.1326 [gr-qc].

[27] Y.S. Myung, Y.-W. Kim and Y.-J. Park, Phys. Lett. B 663, 342 (2008) [arXiv:0802.2152 [hep-th]].

[28] Y. S. Myung, Y. W. Kim and Y. J. Park, Phys. Lett. B 659, 832 (2008) [arXiv:0705.2478 [gr-qc]];

Y. S. Myung, Y. W. Kim, and Y. J. Park, Phys. Rev. D 76, 104045 (2007) [arXiv:0707.1933 [gr-qc]].

[29] Y.S. Myung, Phys. Lett. B 624, 297 (2005) [arXiv:hep-th/0506096].

[30] A. Chamblin, R. Emparan, C. V. Johnson and R. C. Myers, Phys. Rev. D 60, 064018 (1999) [arXiv:hep-th/9902170].

[31] C. Martinez, R. Troncoso and J. Zanelli, Phys. Rev. D 70, 084035 (2004) [arXiv:hep-th/0406111].

[32] Y. S. Myung, Phys. Lett. B 663 (2008) 111 [arXiv:0801.2434 [hep-th]].

[33] R. Yamazaki and D. Ida, Phys. Rev. D 64, 024009 (2001) [arXiv:grqc/0105092].

[34] R. Arnowitt, S. Deser and C. W. Misner, arXiv:gr-qc/0405109.

[35] D. Louis-Martinez, J. Gegenberg and G. Kunstatter, Phys. Lett. B 321, 193 (1994) [arXiv:gr-qc/9309018].

[36] J. Gegenberg, G. Kunstatter and D. Louis-Martinez, Phys. Rev. D 51, 1781 (1995) [arXiv:gr-qc/9408015].

[37] G. Kunstatter, R. Petryk and S. Shelemy, Phys. Rev. D 57, 3537 (1998) [arXiv:gr-qc/9709043].

[38] P.A.M. Dirac, Lectures on Quantum Mechanics", Belfar Graduate School of Sciences (Yeshiva University, New York, 1964).

[39] J. D. Brown and J. W. . York, Phys. Rev. D 47, 1407 (1993). 